\title{
DERECHOS SEXUALES Y REPRODUCTIVOS: UN DEBATE PÚBLICO INSTALADO POR MUJERES
}

\author{
Nélida Bonaccorsi \\ Carmen Reybet
}

Resumen: El propósito del presente artículo es analizar y reflexionar sobre los discursos feministas referentes a sexualidady derechos reproductivos que circulan tanto en el campo teórico como en las normativas dictadas en un ámbito provincial de Argentina. Nos referimos a la Ley sancionada en la provincia de Neuquén, nº 2222/98 sobre derechos sexuales y reproductivos para mujeres y varones.

Palabras clave: Discursos feministas, sexualidad, derechos reproductivos, políticas públicas del estado de Neuquén

Enviado a dictamen: 18 de septiembre de 2008

Aprobación: 17 de octubre de 2008.

Dra. Nélida Bonaccorsi, licenciada en Historia por la UNAM, México; doctora en Estudios de la Mujer, Universidad de Oviedo, España; Facultad de Humanidades, Universidad Nacional del Comahue, Argentina, temas de especialización: Historia cultural y estudios de las mujeres y género, teléfono: 0299-4484433, correo electrónico: nbonacco@calfnet.com.ar.

Mg. Carmen Reybet, licenciada en Antropología por la Universidad Nacional de Rosario, Argentina; Magíster en Ciencias Sociales, Orientación Educación, FLACSO, Argentina; Facultad de Ciencias de la Educación, Universidad Nacional del Comahue, Argentina, temas de especialización: Educación y estudios de las mujeres y género, teléfono: 0299-4473860, correo electrónico: carmen.reybet@speedy.com.ar.
Abstract: The purpose of the present article is to analyze and reflect on the feminist discourses concerning sexuality and reproductive rights, topics that circulate in the theoretical field, as well as in the regulations announced in a provincial environment in Argentina. We are referring to the law pronounced in the Province of Neuquén2222/98 about sexual and reproductive rights towards both men and women.

Key words: Feminist discourses, sexuality, reproductive health, public policies of Neuquén state.

\section{Introducción}

A partir de la re-democratización en la Argentina en los años 80, luego de un largo período de gobiernos de dictaduras militares, el Parlamento comienza a funcionar debatiendo propuestas de leyes impensables de ser sancionadas en otras épocas, y menos aún que favorezcan a las mujeres. Algo similar se produce en las Legislaturas provinciales.

Neuquén, una provincia con menos de medio millón de habitantes, situada al norte de la Patagonia tiene un pasado reciente de defensa continua de los Derechos Humanos por la conformación de organizaciones con ese fin, por una Iglesia progresista y por el grupo local de Madres de Plaza de Mayo. Las organizaciones de mujeres, en esa coyuntura, pudieron constituirse 
y tener un protagonismo decisivo con la propuesta de ley que contempla Derechos de la Mujer, bajo el nombre de "Promoción y garantía de la salud sexual y reproductiva de mujeres y varones", siendo una de las pocas normativas provinciales de esa envergadura con que cuenta el país.

En este artículo nos proponemos presentar los avances teóricos feministas y las experiencias de proyectos que a nivel internacional se han ido articulando sobre el tema, de influencia decisiva en la región, para luego centrarnos en "escribir las prácticas" alrededor del entramado que dio lugar a la pronunciación y sanción de la ley de salud sexual y reproductiva en Neuquén.

\section{El derecho en las mujeres o mujeres con derechos}

A lo largo de la modernidad el campo del Derecho se fue materializando en el dominio masculino / patriarcal, motivo por el cual se constituyó en uno de los espacios de lucha de los movimientos de mujeres que pugnan por instalar en el terreno jurídico la igualdad como horizonte.

Judith Butler y Ernesto Laclau (citado por Faur, 2003) plantean que si bien la noción de igualdad traza un horizonte imposible de ser alcanzado, la tarea política del movimiento de derechos humanos consiste en su búsqueda y en la promoción de su cumplimiento. Estos autores manifiestan que esta tarea política no debe interpretarse como una tendencia hacia la homogeneización de los seres humanos, sino como una aceptación y "proliferación de las diferencias" bajo un común denominador de respeto por sus derechos. Interpretan que, de este modo, en el campo político las nociones de igualdad y diferencia no son incompatibles y que la igualdad es un tipo de discurso que intenta manejar y organizar las diferencias.

La idea de igualdad tiene la capacidad de visibilizar lo diferente de otra manera. Sobre este tema Celia Amorós (2000) expresa que todo derecho a la diferencia supone un derecho a la igualdad, la diferencia en la vida humana es de suyo un hecho, no toda diferencia es buena o mala. Hay diferencias indeseables (por ejemplo, la concepción nazista de la diferencia), hay diferencias neutrales, hay diferencias éticas deseables. Como ha señalado Nancy Fraser, (1991: 26) "toda diferencia que conlleve jerarquía o desigualdad es una mala diferencia". Pues la diferencia es en sí un valor mientras que la igualdad es un concepto regulativo, ético y también es un valor.

Según Alejandra Ciriza (2002), sólo las sociedades que postulan la igualdad - aún cuando formal entre sus ciudadanos-, permiten visibilizar las desigualdades y las diferencias, favoreciendo el nacimiento de movimientos sociales que, como el feminismo, son producto "de la radicalización de la noción de igualdad".

La ciudadanía y los derechos están en continua construcción y cambio, puesto que se conforman con el continuo devenir histórico. Para las mujeres ejercer los derechos de ciudadanía plena es investirse como "sujetos de derechos". En la medida que varones y mujeres ingresan en el mundo de lo jurídico, como ciudadanos de derechos, se enteran que la ley existe y los determina como sujeto social. Así, ambos quedan investidos de la condición de "sujeto de derecho" que significa entrar a la categoría de "ciudadanos".

Al decir de Nancy Fraser (op. cit:: 26) "las necesidades fugitivas adquieren un status político legítimo tendiendo a politizar más esas necesidades". Tratar las demandas de necesidades justificadas como la base para los nuevos derechos sociales es empezar a superar los obstáculos para el ejercicio efectivo del derecho de la ciudadanía y en particular el "derecho de las mujeres". A su vez el reconocimiento de la mujer como sujeto de derecho resignifica el discurso sexista acerca de la mujeres (Ruiz, 2000).

Argumenta Sonia Reverter (2008) que el estado moderno democrático de derecho es en última instancia el garante de los principios de igualdad y justicia, y es el que debe dar un marco para que se evite en la sociedad civil injusticias y desigualdades.

Los movimientos de mujeres sostienen demandas y proclamas tanto específicas de mujeres como igua- 
litaristas, con las cuales se han dirigido al Estado para dialogar, interpelar, hacer propuestas de políticas públicas.

El feminismo tiene presente que el derecho es masculino y patriarcal e ideológicamente opresivo para las mujeres. Si bien es imposible separar el Derecho de la política y de lo ideológico, se trata de que sea inclusivo a todos los integrantes de una sociedad. Para ello se debería des-politizarlo y des-ideologizarlo de su contenido masculinista, permitiendo así que las mujeres puedan acogerse a los beneficios de las leyes. Sólo se trata de pregonar la igualdad del derecho que es sólo el derecho a la igualdad, pero en muchos casos atendiendo las diferencias, y en nuestro estudio, la diferencia sexual particular de las mujeres.

En síntesis, aceptamos la diferencia de los sexos, pero con la igualdad en el derecho, en las oportunidades, en el reconocimiento de la diferencia como fraternidad y sororidad ${ }^{1}$ de los seres humanos.

\section{El cuerpo de la mujer es "privado" o es privado de derechos}

Al hablar del cuerpo nos referimos a la "envoltura del cuerpo" que es analizado desde lo psíquico y social. La representación inconsciente del cuerpo pasa por el imaginario e incorpora elementos de lo simbólico que una cultura construye. Así, la diferencia sexual se distingue de la diferencia biológica entre los sexos. Diferencia sexual como cuerpo: un cuerpo pensante, un cuerpo que habla, que expresa el conflicto psíquico. Es decir, que las diferencias de sexos no son sólo cuestiones anatómicas sino subjetividades, el sexo se asume en el inconsciente independiente de la anatomía² (Lamas, 2000).

A esta concepción de diferencias se le llamó género teniendo en cuenta que las personas son producto de sistemas socio-culturales y que los roles se definen en esos campos simbólicos. Pero la categoría de género ha ido más allá de la diferenciación puramente subjetiva; enmarcó a los sexos en el campo del poder, puesto que no se puede separar la diferencia de la dominación masculina y de la subordinación femenina que es la base del sistema patriarcal. Por lo tanto, la categoría de género es utilizada para discernir, en las relaciones, el poder explícito e implícito que se inflige en toda relación sexual. El cuerpo experimenta distintas sensaciones, dolores, placeres, y la sociedad le impone acuerdos y prácticas psicolegales y coercitivas. El cuerpo se convierte en un nexo peculiar de cultura y elección, y "existir" el propio cuerpo se convierte en una forma personal de asumir y reinterpretar las normas de género recibidas. Judith Butler (2000) lo nombra "cuerpo generizado" como la indistinción de sexo y género subsumidos en la noción de cuerpo.

Los mandatos culturales a las mujeres establecen que su rol natural y social es la reproducción de la especie. A partir de estos preconceptos impuestos, el feminismo contemporáneo denuncia la maternidad obligatoria y la heteronormatividad al proponer repensar los mandatos sociales, a la vez que separar sexualidad de reproducción y objetivar la maternidad como un proyecto de vida, por lo tanto planificada como todo proyecto.

Las instituciones de las sociedades modernas - familia, escuela, iglesia, medios de comunicación, Estado- llegan a convertirse en opresoras de las mujeres por el modo que manipulan la función reproductora. La problemática reside en que se dirime el cuerpo femenino sin la participación de las mujeres en el debate de elección de su propia sexualidad, de sus deseos, pulsiones y metas en la vida. Es necesario que se hable con más claridad desde los distintos espacios de socialización y que se ponga en funcionamiento las prácticas significantes para que el discurso de la sexualidad no quede sólo en el terreno de lo simbólico.

La sexualidad y la maternidad/paternidad son temas de la vida privada-doméstica que deberían ser encarados desde una perspectiva de los derechos humanos. El reclamo por los derechos reproductivos es un reclamo concreto de la práctica de los derechos humanos. Derechos que las personas deben tener en el control sobre el propio cuerpo y deben empoderarse para resistir la imposición 
de conductas no consentidas. El cuerpo de cada una no es del "otro" (persona, institución) sino propio.

La expresión derechos reproductivos alude a una aparente contradicción entre la demanda de autonomía y la demanda de igualdad entre sexos: los derechos reproductivos son los derechos de las mujeres a regular su sexualidad y capacidad reproductora, así como exigir que los hombres asuman responsabilidades por las consecuencias del ejercicio de su propia sexualidad. Por eso es necesario que otros no tengan poder sobre el cuerpo de la mujer y que ésta tenga suficiente consciencia para impedir imposiciones sobre su cuerpo. Esto implica el reconocimiento de derechos humanos básicos, que puede ser interpretado como derecho a la vida, a la libertad, a la prohibición de la esclavitud o de los malos tratos, y todas las formas de violación (marital, por extraños) que son una forma extrema de violencia corporal que tiene significaciones psíquicas. También lo son las imposiciones de métodos anticonceptivos y lo opuesto: la imposibilidad de contar con servicios de salud estatal que aseguren la reproducción consentida (Jelin, 1999).

En la relación con el Estado el dilema que se presenta a la agenda feminista es que, si se aparta de las instituciones estatales por considerar que sus políticas públicas puedan provocar una nueva resubordinación de las mujeres, se corre el riesgo de una pérdida del espacio público que se ha ido conquistando. Por eso mismo, las mujeres no podemos quedarnos fuera de los márgenes que el Estado marca para la acción de la ciudadanía y de la sociedad civil, puesto que desde esos mismos márgenes podremos reestructurar nuevos espacios que incluyan la ciudadanía de las mujeres en plenitud. Según señala Carole Pateman (2000: 208) "se debe ser individuos plenamente autónomos y con derechos en igualdad frente al modelo hasta ahora hegemónico de sujeto varón de derechos".

Los movimientos feministas tradicionalmente han planteado las demandas de las necesidades de las mujeres en general como derechos propios (ley de sufragio femenino, ley contra la violencia doméstica, etcétera) y en la actualidad, hablan públicamente de los hasta entonces innombrables "derechos sexuales" y "derechos reproductivos". ${ }^{3}$ Naturaleza, sexualidad y mujer son categorías que configuran una reelaboración simbólica de las relaciones entre los sexos, en un momento que se hallan en proceso de cambios, por la influencia del pensamiento feminista y del movimiento de mujeres que han alcanzado el ámbito público y reclaman sus derechos, interviniendo continuamente en la elaboración de leyes y políticas públicas que favorezcan a las mujeres.

Los mecanismos del Estado para controlar las identidades y relaciones de género asimétricas están presentes en las modernas democracias. Por ejemplo, las regulaciones en los derechos sexuales llevan como base ideológica una concepción de la mujer-madre y de una sexualidad femenina al servicio exclusivo de la procreación y no del placer, Graciela Di Marco (1999: 205).

Generalmente los grupos o corporaciones que más se oponen a los derechos sexuales son los que hacen alianzas con lo decisores políticos, cuya mayoría se opone al avance de las mujeres, en el sentido que consideran como una amenaza a una "normal vida familiar y sexual".

\section{Notas sobre derechos reproductivos y derechos sexuales}

Los derechosreproductivos hacen referencia a las decisiones y libertades de que un/a sujeto/a debe gozar en orden a decidir sobre sus capacidades reproductivas, al abarcar una gama de derechos que van desde las decisiones acerca de la cantidad y espaciamiento de los hijos, el acceso a servicios adecuados ante situaciones de infertilidad, el acceso a anticonceptivos apropiados; hasta el derecho al aborto seguro, legal y accesible. Los derechos sexuales se refieren más específicamente a la libertad para ejercer plenamente la sexualidad sin peligro de abuso, coerción, violencia o discriminación (Ciriza; 2002). 
Palma señala que aunque el concepto de derechos sexuales es el más inclusivo dado que remite al:

conjunto de derechos inalienables que tienen las personas de tomar decisiones libres y sin coacciones ni discriminación de ningún tipo sobre su propia sexualidad, tanto en sus aspectos corporales y relacionales como en sus aspectos reproductivos; la noción más extendida es la de derechos reproductivos, muchas veces superpuesta con la de salud reproductiva, Zulema Palma (1997: 96).

Y Alejandra Ciriza (op. cit.) ve en el uso generalizado e indistinto de la noción de derechos reproductivos, para hacer referencia a los derechos reproductivos y sexuales, "un síntoma" de las resistencias sociales que la idea de derechos sexuales provoca y que remiten no sólo a la sombra amenazadora de la cuestión del aborto, sino a las imágenes aun más cuestionadoras de orientaciones sexuales diferentes, a la violencia sexual e incluso a la cuestión siempre incómoda de las mujeres en prostitución.

Vistos en perspectiva histórica, los derechos reproductivos han sido definidos antes y mejor que los sexuales, que recientemente han comenzado a debatirse. Por otra parte, lo reproductivo tendió a englobar, subsumir y por lo tanto oscurecer e invisibilizar el campo de lo sexual. No sólo porque así ha sido la tradición y la realidad de la cultura occidental moderna, que hace la ecuación mujer igual a madre; sino porque el mismo campo de la sexualidad se resiste a ser normado.

Los derechos sexuales se instalan en la agenda a partir de las luchas de las mujeres por el acceso a la anticoncepción y al derecho a decidir sobre su propio cuerpo en el marco de la autonomía en las cuestiones sexuales y reproductivas. También gracias al progreso tecnológico que progresivamente permitió separar de manera eficaz la sexualidad de la reproducción, y a partir de las reivindicaciones de los sujetos discriminados como desviados de la normalidad patriarcal, que han buscado ampliar el paradigma de los Derechos Humanos para lograr su inclusión (CONDERS, 2003).

También junto con el paradigma de los derechos humanos, en lasúltimas décadas ha avanzado el reconocimiento cultural y normativo de la diversidad sexual y la lucha por el reconocimiento de nuevas identidades:
La llamada 'identidad sexual' ha dejado de percibirse como estática e inmodificable, para comprenderse como fenómeno que puede cambiar, no solamente a lo largo de la vida, sino en diferentes contextos y situaciones. Se ha comenzado a hablar de identidades fluidas en el contexto de los derechos sexuales. Parte de estos derechos a la libre opción sexual y a no ser discriminado por ello ha comenzado a ser reconocido en las Declaraciones y Planes de acción más recientes, (CONDERS, ibid.: 54).

Los derechos sexuales son un terreno más ambiguo y diverso. Por lo tanto, las principales obligaciones del Estado en esta materia resultan negativas, lo que implica abstenerse de limitar o cercenar tales derechos; para permitir que todos los ejerzan lo más libremente posible sin violar los derechos de los otros (derecho del niño/a a no ser abusado, a no ser casado precozmente, a no realizar prácticas no consentidas).

En síntesis, los derechos sexuales son un campo en construcción.

\section{La agenda feminista en el campo del Derecho}

En la actualidad los distintos instrumentos jurídicos internacionales que comprenden los derechos humanos de las mujeres deben ser vistos como el fruto de un largo proceso de deconstrucción de la trama de sentidos masculinistas incrustados en el derecho liberal desde su etapa fundacional hacia fines siglo XVIII. ${ }^{4}$ Su orientación iusnaturalista, al centrar los "derechos naturales" en la relación del hombre con el Estado, redefine y crea una profunda separación entre los ámbitos público y privado. 
A mediados del siglo XX surge en el seno de la ideología liberal de posguerra el Sistema Moderno de Derechos Humanos, basado en la necesidad de proteger al individuo de las arbitrariedades de los Estados. En ese entonces se produce un giro conceptual: desde los derechos del hombre a los derechos humanos - expresión inclusiva de hombres y mujeres-.

Desde la Organización de Naciones Unidas en el año 1948 se aprueba el primer tratado de aplicación internacional: la Declaración Universal de Derechos Humanos (en adelante DUDH). Como expresa Norberto Bobbio (citado por Faur, op. cit.), la enumeración de derechos que contempla dicha declaración no son ni los únicos ni los posibles derechos humanos, sino los derechos del hombre histórico tal y como se configuraba en la mente de los redactores después de la Segunda Guerra Mundial. ${ }^{5}$ En su opinión, el valor primordial de la DUDH consiste en haber otorgado validez universal a sus declaraciones, lo que supuso "un punto de partida hacia una meta progresiva" que los distintos movimientos sociales a través de sus demandas van redefiniendo y ampliando.

A partir de la firma de la DUDH tuvo lugar un importante proceso de especificación de derechos humanos y de dispositivos de protección, tanto regional como internacional. Además de los mecanismos orientados a establecer sistemas generales de protección, aparecieron otros destinados a proteger ciertas categorías de personas: mujeres, niños, trabajadores, refugiados, discapacitados, etcétera, o a proteger ciertas ofensas singularmente graves contra los derechos humanos, como el genocidio, la discriminación racial, el apartheid, la tortura o la trata de personas.

Las áreas contempladas han experimentado un proceso gradual de ampliación, en forma paralela al cambio de paradigma en el que se basan. Por ello puede afirmarse que, aún en su debilidad, el sistema moderno de Derechos Humanos es una herramienta de suma utilidad al movimiento de mujeres mundial y que representa significativos avances en sus reivindicaciones al otor- garles legitimidad simbólica. Hoy los derechos humanos de las mujeres se han extendido considerablemente al abarcar ámbitos anteriormente considerados privados, como el de la violencia doméstica. Otra de las recientes áreas ha sido la de la salud sexual y reproductiva, objeto de nuestro análisis.

\section{Proceso de especificación de los Derechos Huma- nos de las Mujeres: de la CEDAW a Beijing}

A partir de la llamada Década de la Mujer (1975-1985), los derechos ciudadanos para las mujeres han conocido una significativa expansión hasta el punto de marcar un hito tanto en la historia del movimiento de mujeres como en la teoría feminista. Empowerment ${ }^{6}$ y entitlement, ${ }^{7}$ parecen ser las palabras para caracterizar esta fase de la historia del movimiento de mujeres y del feminismo (Ciriza: op. cit.).

El examen de los documentos producidos en las distintas cumbres internacionales de Naciones Unidas desde la Convención por la Eliminación de todas las Formas de Discriminación contra la Mujer (en adelante CEDAW, 1979), permite registrar dicha expansión. Las Plataformas de Acción de las siguientes Cumbres Internacionales: Conferencia Mundial sobre Derechos Humanos (Viena, 1993), Conferencia sobre Población y Desarrollo (El Cairo, 1994), y la IV Conferencia Internacional sobre la Mujer (Beijing, 1995) registran sucesivos cambios en la orientación del análisis y en las recomendaciones.

La CEDAW amplía la Declaración Universal de los Derechos Humanos, procurando orientar disposiciones para alcanzar la igualdad entre hombres y mujeres en distintas esferas sociales. Sus postulados, centrados en áreas como la salud, la educación, la justicia, el trabajo y la participación política, especifican las medidas necesarias para eliminar la discriminación basada en el género. A partir de su firma el Derecho - o parte de él- tuvo la oportunidad de operar como un elemento transformador de las disparidades de género en tanto 
los Estados participantes asumen como injusticia la existencia de muchas de las disparidades de género y se comprometen a: otorgar un trato igualitario para hombres y mujeres, sancionar cualquier tipo de práctica que perpetúe esa desigualdad, y promover medidas de acción positiva de carácter temporal para su transformación (Faur, op. cit.).

A modo de balance crítico señalamos que si bien en uno de sus considerandos se expresa la existencia de discriminación contra las mujeres; el modelo sigue siendo el de la DUDH, con lo cual la CEDAW se encuadra en la división público/privado y la gran mayoría de sus artículos refiere a actividades de las mujeres en el ámbito público, no incorporando el ámbito de lo privado (Navarro, 1994).

En Argentina, el debate por los derechos de las mujeres se inició a partir de la restauración democrática, bajo la presión de condiciones internacionales que legitimaba los derechos de las mujeres. En 1985 el gobierno democrático de Raúl Alfonsín suscribió la CEDAW, en un complejo escenario marcado no sólo por la presencia del tema en el nivel internacional, sino por la asunción de un rol activo por parte del Estado, y por las presiones desiguales y contradictorias ejercidas por diferentes sectores de la sociedad civil, desde el movimiento de mujeres cada vez más complejo y fragmentado, ${ }^{8}$ hasta la poderosa Iglesia Católica argentina (Ciriza, op.cit.).

La IV Conferencia de Naciones Unidas sobre la Mujer, realizada en Beijing, representa un hito para el movimiento de mujeres en tanto recoge en gran medida las propuestas que el movimiento feminista universal venía enarbolando. A lo largo del Proceso Beijing (1993-1995) que incluye la preparación, ${ }^{9}$ realización y seguimiento, se debatió una agenda propia de las mujeres, y las delegaciones de los gobiernos participantes aprobaron una extensa plataforma de acción. Esta Conferencia dio tratamiento a los Derechos Sexuales y Reproductivos como Derechos Humanos, y no como políticas demográficas (restrictivas o pro-natalistas). El Párrafo 96 de la Plataforma de Acción expresa:
Los Derechos Humanos de la mujer incluyen el derecho a tener control y decidir responsablemente sobre los asuntos relativos a su sexualidad, incluidas su salud sexual y reproductiva, libres de coerción, discriminación y violencia. ${ }^{10}$

Producto de la compleja trama de relaciones que se fue tejiendo entre el movimiento de mujeres y los gobiernos a nivel nacional, regional e internacional, los avances fueron posibles, entre otros factores, por la creación de un "triángulo de empoderamiento" que articuló una alianza entre feministas de la sociedad civil y el estado en distintas posiciones de poder, que adquirió un contenido concreto y flexible.

En Argentina, a partir de la década de los 90, la cuestión de los derechos reproductivos y sexuales se convirtió en objeto de políticas, legislaciones y debates públicos. La presentación y sanción de proyectos de leyes conforma un listado variado y heterogéneo en cuanto a sus alcances y fundamentos, mostrando hasta qué punto los derechos reproductivos pueden ser considerados como un punto de desacuerdo y conflicto entre el Estado, la sociedad civil y el movimiento feminista y de mujeres. ${ }^{\text {Il }}$ Por lo general, puede afirmarse que la problemática de la salud y los derechos sexuales y reproductivos se encuentra en el pasaje desde una situación homogéneamente restrictiva - propia de las décadas precedentes - a otra en la que van produciéndose larvados cambios y aperturas, que de ningún modo siguen una trayectoria lineal. ${ }^{12}$ En distintas jurisdicciones provinciales y municipales se ha impulsado, con suerte dispar, el tratamiento y/o sanción de proyectos de ley ${ }^{13}$ representan un punto de inflexión respecto a la tradicional actitud refractaria del Estado respecto a la regulación de la fecundidad asentada en argumentos pronatalistas, ${ }^{14}$ los que permeaban tanto las políticas como los programas de salud (Ramos, 2000).

La feminista neuquina Andrea Diez (2000) sostiene que el reclamo de las organizaciones argentinas de mujeres se produjo — con diferencias y matices muy 
diversos-, sobre dos líneas específicas: por un lado la ubicación de los derechos sexuales y reproductivos como derechos humanos, enfoque que incluye el derecho a lograr una mejor calidad de vida para las mujeres evitando muertes y enfermedades; lo que requiere concebir a la mujer como sujeto - y no objeto- de las políticas públicas. Por otro lado, la introducción de cambios necesarios que hagan posible un ejercicio efectivo de la autonomía sexual y reproductiva de las mujeres, lo que implica necesariamente un cuestionamiento de las relaciones intergenéricas.

Tal como lo indican Teresa Durand y María Alicia Gutiérrez (1998), la lucha de las mujeres en América Latina por decidir sobre su propio cuerpo está, en nuestro siglo, ligada a dos inflexiones históricas: la de los 60 y $70,{ }^{15}$ y la de los 80 y 90, marcada tanto por las estrategias defensivas ante el avance neoconservador, como por los efectos de la restauración democrática, en el sentido de la expansión de derechos formales y la creación de espacios estatales para la implementación de políticas hacia las mujeres, y, en el caso argentino, por una profunda transformación tanto cuantitativa como cualitativa en el movimiento de mujeres y en el feminismo.

\section{Provincia de Neuquén: el papel del movimiento local de mujeres en el proceso de sanción de una ley sobre salud sexual y reproductiva}

El contexto provincial en el que se enmarca la promulgación de la ley 2222/97 presenta características peculiares que se remontan a la década del 60. A mediados de dicha década, desde el nivel nacional se formaliza el Modelo de Programación de Actividades de Salud en el contexto del Programa Sanitario Preliminar de Acción contra la tuberculosis, hidatidosis y mortalidad infantil con directivas precisas del gobierno nacional para que se concreten en cada provincia. Este Programa de interacción entre Nación y provincias fue la base —vista a la distancia, su primera etapa - sobre la que se edificó en la década del 70 el Plan Integral de Salud neuquino, plan que carece de analogía en la historia argentina y aún hoy en día se convierte en un modelo de referencia para toda Latinoamérica.

Entre algunos factores que permiten dar cuenta del origen de este paradigmático Plan provincial cabe señalar la multiplicación y heterogeneidad de la población, incrementada especialmente por inmigrantes ${ }^{16}$ provenientes del interior del país y de Chile, atraídos por una próspera economía incipiente producto de la explotación petrolífera y gasífera, la producción frutícula, ganadera ovina y la consecuente proliferación del comercio y los servicios. Estas peculiaridades de la provincia le permitieron experimentar, entre las décadas de 1970 y 1980, un vertiginoso ritmo de crecimiento que duplicó su población — a la fecha alcanza unos 450 mil habitantes-. A su vez, en el aspecto político, tuvo lugar la conformación de un partido provincial: el Movimiento Popular Neuquino que, en su etapa constitutiva de los años 60, atrajo en su seno a sectores progresistas.

En este marco, la programación del Plan Integral de Salud fue realizada por prestigiosos/as profesionales de la salud provenientes de la Capital Federal ${ }^{17}$ quienes visualizaron que estaban dadas las condiciones — prosperidad económica y apoyo político - para diseñar eimplementar prácticas sanitarias pensadas desde una "mística igualitaria, equitativa, con justicia social". Esta singularidad amplía el sentido de la oferta en salud al ser también abordada como "una oferta política, una oferta cultural, una oferta espacial" (Villarruel y Morate, 1990: 4). ${ }^{18}$

La conformación de una masa crítica de sectores medios con ideas progresistas ha teñido hasta el día de hoy la vida cultural de la sociedad neuquina que, durante los años de laúltima dictadura militar (1976-1983) y bajo el liderazgo del Obispo Jaime de Nevares colocó a la provincia en un férreo lugar de defensa de los derechos humanosy, desde la re-democratización del país, se expresó en el surgimiento de distintas agrupaciones sociales constituidas en torno a la defensa de los derechos humanos, de los pueblos originarios, de los derechos de las mujeres, de los derechos de la niñez y la adolescencia, entre otros. 
Hacia la década del 90, con relación a nuestro tema de análisis, se destacan en Neuquén dos organizaciones feministas - "Condición femenina” y "Mujeres por el derecho a elegir"- que, aún con escaso número de adeptas (menos de 20 miembros) desempeñaron en el contexto post-Beijing un importante papel de sensibilización y promoción de los derechos de las mujeres. Junto a mujeres trabajadoras del sector público provincial integraron una Multisectorial que permitió alumbrar en el año 1997 una ley de salud sexual y reproductiva, ley 2222/97 aprobada por unanimidad en la legislatura provincial.

Una característica notable de esta ley es que su contenido refleja las recomendaciones emanadas de las últimas Conferencias Internacionales: sobre Población y Desarrollo en El Cairo (1994) y sobre la Mujer en Beijing (1995). Algunos de sus objetivos son: reducir la tasa de morbi-mortalidad materno-infantil; tender a la disminución de las enfermedades de transmisión sexual de mujeres y de varones; establecer políticas de prevención y atención en la salud sexual y reproductiva de los y las adolescentes; ofrecer información y asesoramiento sobre los métodos de anticoncepción disponibles, su efectividad, contraindicaciones, ventajas y desventajas; prescripción, colocación y/o suministro de anticonceptivos; controles de salud, estudios previos y posteriores; capacitación sistemática de los equipos interdisciplinarios involucrados a fin de promover, prevenir e informar sobre los aspectos relacionados con la salud reproductiva.

Andrea Diez ${ }^{19}$ repasa el proceso de participación del movimiento de mujeres de la provincia en torno a la elaboración y presentación del anteproyecto. Entre otros aspectos de interés, reflexiona sobre las dificultades de nombrar el cuerpo deseante de las mujeres y sobre los riesgos que se asume al optar por el término "salud reproductiva" o "derechos reproductivos", sin incluir sus pares: "salud sexual" y "derechos sexuales".

En su relato expresa que la iniciativa estuvo urgida por la necesidad de "salirle al cruce" a un Proyecto de ley que había sido presentado por un diputado mé- dico sobre Planificación Familiar Voluntaria "que no incorporaba los nuevos paradigmas del movimiento de mujeres legitimados por las plataformas de Viena, Cairo y Beijing".

Narra que en el anteproyecto alternativo, las autoras concientemente eludieron usar el término "salud sexual", como un modo de prever eventuales resistencias de los/ as diputados/as provinciales. Esa omisión, a la distancia, le hace decir: "un anteproyecto que había partido de la premisa de nombrar nuestros cuerpos sexuados para garantizarle derechos, había quedado atrapado en las significaciones sociales de mujer-reproducción". Y se cuestiona: "¿Acaso lo reproductivo silencia lo sexual?", pregunta que le suscita las siguientes reflexiones. La primera de ellas apunta a las dificultades de usar sólo la expresión del término "salud reproductiva", de alcance más restrictivo frente al de "salud sexual".

Desprendemos de su análisis, la existencia de una tensión entre dos lógicas: la de "la praxis feminista" según la cual las leyes de salud reproductiva inmediatamente implican las dimensiones de autonomía sexual y reproductiva de las mujeres; y la de otras redes discursivas - no feministas - que parecen no decodificar de la misma forma, al menos en el primer impacto.

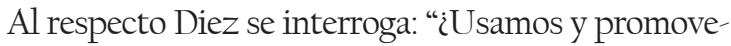
mos el término salud reproductiva porque está tomado en su amplia concepción que no sólo incluye la reproducción, o porque resulta menos lesivo y menos provocador a la hora de nombrar y legitimar públicamente nuestros derechos sexuales? Y, en esta línea, iqué efecto termina produciendo este uso político, en el proceso de lobby y consenso para la obtención de leyes? Y ¿qué efecto produce posteriormente a la hora de hacer cumplir esas leyes?".

En su relato basado en la experiencia, Diez expresa que el anteproyecto recaló en la Comisión de Salud, primer ámbito de discusión en el que las autoras iniciaron su tarea política de convencimiento de los/as diputados sobre la necesidad de contar con una ley que legitime el derecho a decidir en el campo sexual. En ese espacio legislativo, el título 
acordado inicialmente fue el de salud reproductiva. Paradojalmente fue una de las diputadas quien, ante la naturaleza de los argumentos esgrimidos por las mujeres de la Multisectorial — profusos en el señalamiento de situaciones de inequidades de género-, reaccionó declarando: "Entonces esta ley no se va a llamar sólo de Salud Reproductiva, sino de Salud Sexual y Reproductiva".

Acentuamos que las diputadas de la citada Comisión - pertenecientes a distintas extracciones políticasfueron las más receptivas al planteo efectuado y además las promotoras de persuadir a sus compañeros/as de bancada.

Como dato cabe señalar que tanto el diputado que presentó el primer anteproyecto de ley, como dos de las legisladoras son de profesión médica. Situación ésta que tiñe los términos y los contenidos de la ley aprobada, que pone el acento en la salud reproductiva.

Respecto al debate plenario sobre el tratamiento de la ley salud sexual y reproductiva en la sesión de la Legislatura, se comenta que el mismo fue de corta duración. Algunos de los fragmentos de la sesión de la Cámara de Diputados, evidencian la preocupación por: "Disminuir los embarazos adolescentes y de abortos provocados con la consiguiente secuela de -morbi-mortalidad materna"; "Muchos hogares se ven golpeados a raíz de esta estadística (de embarazos adolescentes). Pero no es el golpe sólo al hogar, es el golpe a la madre adolescente y al niño que viene al mundo y no fue deseado".

Sin embargo, ante estos argumentos, una diputada con registro feminista, fue más allá de lo meramente llamado salud reproductiva y planteó:

...creemos que la sociedad argentina está madura hasta ahora, lo mismo la neuquina, para consensuar todo lo que hace a la protección de la sexualidad y la reproducción. No creemos que la sociedad argentina esté para consensuar, por ejemplo como otros países lo hacen, una ley de despenalización del aborto.
En esta última intervención asoma el indicio del solapamiento de una cuestión que no se ha debatido en los ámbitos estatales. Trascurridos once años de la sanción de la ley provincial, tanto en el territorio nacional como regional, el aborto no ha logrado imponerse como tema de la agenda pública. Muchas son las causas de este silenciamiento, fundamentalmente la fuerte presencia de la iglesia católica.

Como conclusión nos interesa realizar un aporte crítico a la letra de la ley. La misma enfatiza los derechos reproductivos dejando de lado la promoción de los derechos sexuales tanto de mujeres como de varones, corroborando así los señalamientos que hemos desarrollado a lo largo del artículo sobre la base de los planteos teóricos feministas. Recordamos que los derechos sexuales son un campo en construcción que requiere del aporte de los movimientos de mujeres y otros colectivos, en pos de trascender los límites y ampliar las posibilidades de los derechos reproductivos.

\section{Notas}

${ }^{1}$ Significa hermandad entre mujeres; viene del latín sor, hermana.

2 Si bien este es un planteamiento de las feministas psicoanalíticas, es aceptado por las demás corrientes del feminismo.

${ }^{3}$ Hablar de derechos sexuales nos insta a profundizar sobre los discursos, las ideas filosóficas, mitos y creencias sobre la sexualidad (Puleo, 2002).

${ }^{4}$ Así, la Declaración de los Derechos del Hombre y del Ciudadano de 1789 representa un primer paso en la noción de derechos civiles y políticos, aunque todavía muy restringido, dada las distintas marcas de exclusión que porta: todas las mujeres y niños; algunos hombres.

${ }^{5}$ Esta afirmación significa entender que los Derechos Humanos son fruto de un proceso histórico, contra la teoría iusnaturalista que asume que los Derechos $\mathrm{Hu}$ manos son inherentes a la "naturaleza humana". 
${ }^{6}$ El concepto implica desafiar la dominación masculina y la subordinación femenina, transformando las estructuras y las instituciones que refuerzan y perpetúan las discriminaciones de género y las desigualdades sociales, al tiempo que posibilitan que las mujeres tengan acceso y control sobre los recursos materiales y la información (Durand y Gutiérrez; 1998).

${ }^{7}$ Entitlement remite a la definición de titularidad de los derechos (ibíd.).

${ }^{8}$ En el caso argentino el movimiento de mujeres no sólo está constituido por feministas, sino por mujeres no feministas: mujeres de sectores populares en lucha por la subsistencia, grupos no menores del movimiento por los derechos humanos y minorías sexuales: travestis, transexuales, gays, lesbianas, bisexuales (Ciriza, op.cit.).

${ }^{9}$ En la Región cabe señalar la realización de la Conferencia Regional de América Latina y el Caribe (Mar del Plata, 1994).

${ }^{10}$ Es un avance con relación a la plataforma de la Conferencia sobre Población y Desarrollo de El Cairo efectuada un año anterior, que incluye la sexualidad dentro de la salud reproductiva.

${ }^{11}$ Como referencia, algunos hitos de los debates públicos a propósito de los derechos reproductivos y sexuales de las mujeres vinculados a dos acontecimientos relevantes en el orden internacional: la Conferencia sobre Población y Desarrollo (El Cairo, 1994) y la IV Conferencia Internacional sobre la Mujer (Beijing, 1995). El gobierno argentino ha sido en este punto sumamente coherente. Durante los diez años de gobierno constitucional de Carlos Menem las delegaciones oficiales argentinas no sólo han intentado obstaculizar la posibilidad de establecer acuerdos en favor de la despenalización del aborto en el nivel internacional, haciendo reservas de manera sistemática, sino que, en el frente interno el gobierno intentó sabotear de diversas maneras los avances legales en materia de derechos sexuales y reproductivos.

${ }^{12}$ El advenimiento del régimen democrático en el año 1983 conllevó la derogación de las normas restrictivas del control de la natalidad que establecían medidas coercitivas sobre la venta y comercialización de anticonceptivos, pero sin que ello supusiera la inmediata y generalizada implementación de acciones positivas (Ramos, 2000).

${ }^{13}$ Han sido pioneras: las provincias de Río Negro, La Pampa, Chaco, Córdoba, Mendoza y Neuquén; y los municipios de Córdoba, Rosario y la Ciudad Autónoma de Buenos Aires. Con posterioridad, en el año 2002 se sancionó la ley nacional № 25.673 de Salud sexual y procreación responsable.

${ }^{14}$ Sustentadas en el lento crecimiento demográfico y/o en consideraciones de orden geopolítico (Ramos, ibíd.).

${ }^{15}$ Cuya impronta está dada por las rebeliones juveniles y la irrupción de un cambio cultural sin precedentes en el mundo occidental.

${ }^{16} \mathrm{El}$ aporte migratorio se divide fundamentalmente en dos sectores poblacionales: profesionales y técnicos de distintas ramas, y trabajadores/as de servicios.

${ }^{17}$ Ciudad de Buenos Aires.

18 Entre los objetivos señalados en el Plan de Salud tanto de zonas rurales como urbanas algunos eran: 1 . Realizar la atención del parto en los establecimientos hospitalarios. 2. Controlar a las embarazadas según normas de las Pautas de Programación para Atención Médica Rural (PPAMR). 3. Controlar a los niños menores de 5 años PPAMR. 4. Prestar atención alimenticia a embarazadas y niños menores de dos años. 5 . Controlar a los escolares concurrentes de las zonas I, II, II y IV. El control será efectuado por médico en la misma escuela o por citación de alumnos al establecimiento según la proximidad entre escuelas y servicio. 6. Prevenir las enfermedades trasmisibles evitables por vacunaciones. 7. Detección, control y tratamiento de tuberculosis y sus contactos. 8. Educación sanitaria realizada por Agente Sanitario a través de las rondas. 9. Adecuar los establecimientos del área de programación para satisfacer la demanda promovida por las actividades del programa. 10. Aproximar los actuales niveles de complejidad de los establecimientos a los 
modelos correspondientes según guía de clasificación de los servicios de Atención Médica.

${ }^{19}$ Integrante de la ONG local "Mujeres por el Derecho a Elegir".

\section{Bibliografía}

Amorós, Celia, 2000, "Elogio de la vindicación”, en Alicia Ruiz (comp.), Identidad femenina y discurso jurídico, Ed. Biblos, Buenos Aires, pp. 51-63.

Bonaccorsi, Nélida, 2002, Políticas Públicas de Igualdad, Universidad de Oviedo, España, Universidad Nacional del Comahue, Ed. PubliFadecs, Roca, Argentina.

_, 2003, "Las prácticas discursivas sobre salud sexual: a propósito de las políticas públicas del Estado neuquino" en La Aljaba, segunda época, Revista de Estudios de la Mujer, vol. VIII, Editan Universidades de Luján, La Pampa y Comahue, ciudad de Santa Rosa, Argentina, pp. 209-220.

Bourdieu, Pierre, 2000, La dominación masculina, Barcelona, Anagrama.

Butler, Judith, 2000, "Meramente cultural", en New leftreview, núm. 2, (versión en español), Ed. Akal, Madrid, pp. 109-121.

Ciriza, Alejandra, 2002, "Consenso y desacuerdo. Los derechos reproductivos como derechos ciudadanos de las mujeres en Argentina", en El Catoblepas, Revista Crítica del Presente, núm. 9, en www.nodulo.org.

Diez, Andrea, 2000, "Nuevos dilemas iviejos discursos?", (Ponencia), Seminario de Salud Reproductiva en la esfera pública y política de América Latina y el Caribe, Consorcio Nepo-Unicamp. Brasil.

CONDERS (Consorcio Nacional de Derechos reproductivos y Sexuales), 2003, "Situación de la Atención de la Salud sexual y reproductiva desde la perspectiva de las/os usuarias/os", Buenos Aires. (Documento).

Di Marco, Graciela, 1991, “Ciudadanía femenina”, en Relaciones de género y exclusión en la Argentina, Primeras Jornadas de ADEUM, Ed. Espacio, Buenos Aires, pp. 204-213
Durand, Teresa y Alicia Gutiérrez, 1998, "Tras las huellas de un porvenir incierto: del aborto a los derechos sexuales y reproductivos", en Avances en la Investigación Social en Salud Reproductiva y Sexualidad, Eds. AEPA, CEDES, CENEP, Buenos Aires, pp. 281-302.

Faur, Leonor, 2003, “iEscrito en el cuerpo? Género y derechos humanos en la adolescencia", en Susana Checa (comp.), Género, sexualidad y derechos reproductivos en la adolescencia, Ed. Paidós, Buenos Aires, pp. 37-75.

Fraser, Nancy, 1991, "Las luchas por las necesidades", en Debatefeminista, año 2, vol. 3, México, pp. 3-40.

Hernández, Adriana, Carmen Reybet y Nélida Bonaccorsi, 2002, "De lo internacional a lo regional: Ley de Salud Sexual y Reproductiva-2222/97- y desafíos en educación", ponencia presentada ante Jornadas $\mathrm{Na}$ cionales La región, un ámbito para la planificación y la acción, Universidad Nacional del Comahue, Neuquén.

Hernández, Adriana, Carmen Reybet y Andrea Diez, 2001, "Ley Provincial de Salud Sexual y Reproductiva: La escuela interpelada. Indicios para la reconstrucción de una trama discursiva escolar acerca de la sexualidad", en Actas Pedagógicas año 2, núm. 1, Ediciones Manuscritos, Facultad Ciencias de la Educación Universidad Nacional del Comahue, pp. 42-5l.

Lamas, Marta, 2000, "Género, diferencia de sexo y diferencia sexual”, en Alicia Ruiz (comp.), Identidad femenina y discurso jurídico, op. cit. pp. 65-84.

Navarro, Marysa, 1994, "Los derechos humanos de las mujeres”, en Debate feminista. año 5, vol. 10, México, pp. $217-224$

Palma, Zulema, 1998, "El aborto y los derechos sexuales desde el movimiento de mujeres en el contexto de la Argentina democrática”, en Nuestros cuerpos, nuestras vidas: propuestas para la promoción de los derechos sexuales y reproductivos, foro por los derechos reproductivos, Buenos Aires, pp. 95-102.

Pateman, Carole, 2000, "Feminismo y democracia", en Marisa Navarro y Catharine Stimpson (comp.), Cambios sociales, económicos y culturales, Ed. Fondo de Cultura Económica, Buenos Aires, pp. 189-210. 
Puleo, Alicia, 2002, "Filosofía, política y sexualidad", en María Luisa Femeninas (comp.), Perfiles del feminismo iberoamericano, Buenos Aires, Ed. Catálogo, Buenos Aires, pp. 303-316.

Ramos, Silvina, 2000, Presentación Taller-Seminario salud sexual y reproductiva de la provincia de Mendoza, CEDES, Buenos Aires.

Reverter, Sonia, 2008, "Ciudadanía, sociedad civil y género", en revista La Aljaba, segunda época, Revista de Estudios de la Mujer, núm. 12, Ed. Universidad Nacional del Comahue, (EDUCO), Neuquén, Argentina (en prensa).

Reybet, Carmen y Mónica Oppezzi, 2007, "Estrategias institucionales a escala local frente al embarazo adolescente: el caso de una escuela media y un centro de salud de la ciudad de Neuquén", en Elsa López y Edith Pantelides (comp.), Aportes a la investigación social en salud sexual y reproductiva, Eds. CENEP, CEDES, AEPA, UNFPA, Buenos Aires, pp. 331-362.

Romero, Teresa y Laura Quilodrán, 2008, “Acerca de los derechos sexuales y reproductivos. Ley neuquina 2222/97", en Trabajo monográfico presentado al Seminario Ciudadanía y Políticas Públicas para las Mujeres, Especialización en Estudios de las Mujeres y de Género, Universidad Nacional del Comahue.

Ruiz, Alicia, 2000, "La construcción jurídica de la subjetividad no es ajena a las mujeres", en Haydeé Birgin, El Derecho en el género y el género en el Derecho, Ed. Biblos, Buenos Aires, pp. 107-120.

Villarruel, José y Morate, Ada (comps.), 1990, Estado, sociedad, salud: Neuquén (1944-1972), Investigación histórica sobre el Plan de Salud Neuquino, Ministerio de Salud pública, Universidad Nacional del Comahue. 